\title{
Validade concorrente da escala de conforto para familiares de pessoas em estado crítico de saúde (ECONF) e o Self-Reporting Questionnaire (SRQ-20)
}

\author{
Ednaldo Magalhães Ferreira Filhoㅜ; Kátia Santana Freitas²; João Victor Moraes \\ de Melo ${ }^{3}$ \\ 1. Bolsista PIBIC/CNPq, Graduando em Medicina, Universidade Estadual de Feira de Santana, e-mail: \\ ed.2455@gmail.com \\ 2. Orientador, Profa Adjunto B do Departamento de Saúde, Universidade Estadual de Feira de Santana, e-mail: \\ freitaskatia@yahoo.com.br \\ 3. Voluntário do Núcleo Interdisciplinar de pesquisa e estudos em Saúde (NIPES), Graduando em Medicina, \\ Universidade Estadual de Feira de Santana, e-mail: joaomoraesmelo@ hotmail.com
}

PALAVRAS-CHAVE: ansiedade; família; cuidados de conforto

\section{INTRODUÇÃO}

A internação em UTI gera um alto grau de estresse e ansiedade nas famílias devido muitas vezes a impossibilidade em se comunicar e principalmente pela gravidade clínica do paciente. Tais fatores podem interferir na capacidade dos familiares de desempenhar o papel de cuidador e também pode dificultar suas tarefas diárias (Jezierska et al, 2014).

A UTI caracteriza-se como uma unidade complexa dotada de sistema de monitorização contínua, que admite pacientes potencialmente graves ou com descompensação e que, com suporte e tratamento intensivos, aumenta a possibilidade de recuperação. Para os familiares essa experiência é percebida, muitas vezes, como agressiva e angustiante, requerendo dos profissionais uma comunicação clara e constante com as famílias a fim de reduzir o descontrole emocional diante do enfrentamento da possibilidade do rompimento do vínculo familiar (Puggina, 2014). Nesse momento, a atenção da família, está centrada na condição de ameaça à vida de seu parente e na sua evolução, e isto pode ocasionar um desconforto para com elas mesmas, como não se alimentar, não conseguir descansar, postergando suas necessidades e seus problemas de saúde (Freitas, 2012).

Em muitos momentos a família se sente impotente, aborrecida, inconsolável, solitária, triste e, culpada por não poder cuidar do seu familiar. O enfrentamento da crise provocada pela hospitalização pode provocar alterações psicológicas evidenciadas através de estados de angústia, ansiedade, medo, insegurança e depressão (Moreira; Martins; Castro, 2012).

Foi constatado que risco do familiar de paciente internado na UTIs desenvolver a Síndrome de estresse agudo e Transtorno de estresse pós-traumático (Stress Syndrome and Posttraumatic Stress Disorder- PTSD) varia de 33-49\%, enquanto que o risco de depressão é estimada em $6 \%$ a $26 \%$, dependendo do momento do estudo. Já o risco estimado de PTSD em famílias cujo o parente faleceu na UTI é de $14-56 \%$ e o risco de depressão é de 18-42\%, incluindo transtornos depressivos graves cuja incidência é de $27 \%$ (Jezierska et al, 2014).

Diante do exposto sobre a hospitalização de um familiar em uma UTI, condição que leva a família a vivenciar situações de desconforto, reconhece-se a importância da adequada aferição do construto conforto no contexto brasileiro. Frente a esse panorama, Freitas (2012) construiu um instrumento que permite a avaliação do nível de conforto vivido por familiares no contexto da unidade de terapia intensiva, denominado Escala de Conforto para Familiares de Pessoas em Estado Crítico de Saúde (ECONF). Assim, 
tendo em vista a importância de se utilizar instrumentos de medida validos propõe-se como objetivo avaliar a validade concorrente da ECONF a partir de um critério externo.

Nesse estudo o instrumento utilizado como critério foi o Self-Reporting Questionnaire (SRQ-20) que foi desenvolvido, na década de 1970, pela Organização Mundial de Saúde (OMS) com propósito de avaliar os impactos dos transtornos mentais comuns (TMC) na atenção básica em países em desenvolvimento. Os TMC são sintomas não psicóticos, que podem ser insônia, fadiga, irritabilidade, esquecimento, dificuldade de concentração e queixas somáticas.Atualmente na literatura científica, observou uma variabilidade da especificidade em pesquisas internacionais de $44 \% 19$ a $95,2 \%$, enquanto entre estudos nacionais verificou-se de $74,6 \%$ a $89,3 \%$; já a sensibilidade variou de $73 \% 5$ a $89,7 \%$ e de $57 \%$ a $86,3 \%$, respectivamente para pesquisas fora do país e no país (Santos et al, 2011).

Este estudo poderá contribuir para o fortalecimento da Escala de conforto de familiares como medida válida, estabelecer uma relação entre as duas escalas e bem como identificar o nível de conforto e as alterações psíquicas desenvolvidas por familiares decorrente a hospitalização de um ente. Tal conhecimento contribui para fomentar a discussão sobre o tema e suas possibilidades de intervenção pelos profissionais de saúde com intuito de minimizar os desconfortos de ordem psíquica e emocional vividos por estes indivíduos.

\section{MATERIAL E MÉTODOS}

Este plano de trabalho está inserido no projeto Construção e validação de uma escala de conforto para familiares de pessoas em estado crítico de saúde (ECONF) cujo escopo é reforçar as evidências de validade e aplicação da ECONF para a avaliação do nível de conforto de familiares de Pessoas em estado crítico de saúde.

Trata-se de um estudo transversal realizado em duas Unidades de terapia intensiva geral de um hospital público de grande porte, no município de Feira de Santana. O projeto obteve aprovação pela Comissão de Ética para Análise em Projetos de Pesquisa do Hospital Universitário Professor Edgar Santos, mediante o Parecer 078/09.

A população foi constituída de 100 familiares de pessoas adultas internadas nas UTI de um hospital público de Feira de Santana - BA, que atenderam aos seguintes critérios de inclusão: ter idade igual ou superior a 19 anos; ser a pessoa mais próxima do parente hospitalizado, que com ele convive e mantém relacionamento estreito; ter um familiar adulto internado na UTI com mais de $48 \mathrm{~h}$ de internação; ter realizado pelo menos uma visita ao parente. A amostragem foi por acessibilidade, incluídos no estudo aqueles indivíduos que aceitaram participar e atenderam aos critérios de elegibilidade.

Os dados sociodemográficos e clínicos, assim como a aplicação da ECONF e SRQ-20, foram obtidos por meio de entrevista.

Foi utilizada a ECONF para avaliação do nível de conforto, uma vez que se trata de uma escala para medida de conforto validada. A ECONF é constituída de em três dimensões: Segurança (20 itens), Suporte (20 itens) e, Interação familiar e ente (6 itens). A escala de medida é crescente, ou seja, quanto maior o valor atribuído aos itens, maior é o grau conforto. Sendo a pontuação: 1- Nada confortável, 2 - Pouco confortável, 3 Mais ou menos confortável, 4 - Muito confortável e 5 - Totalmente confortável (Freitas, Menezes, Mussi, 2015).

O SRQ-20 é um instrumento utilizado para a suspeição diagnóstica dos transtornos mentais comuns (Santos; Araújo; Oliveira, 2009). É composto por 20 itens distribuídos em quatro dimensões, destina-se a detecção de sintomas não psicóticos (Santos et al., 2011). Para cada uma das suas questões contém uma escala dicotômica sim/não, pode ser respondido através do autopreenchimento ou entrevista. O ponto de 
corte atribuído para casos positivos foi maior ou igual a 7 para rastreamento positivo para transtorno mental comum.

Para a análise da validade de critério, foi empregado o coeficiente de Correlação de Pearson. O nível de significância estatística adotado foi de 5\%. Para análise das variáveis categóricas foi utilizada a estatística descritiva, como frequências absoluta e relativa. Para as variáveis quantitativas foram calculadas as medidas descritivas de centralidade (média, mediana e moda) e de dispersão (desvio- padrão).

Foram calculados os escores das escalas como um todo e de suas respectivas dimensões. Após a coleta dos dados, esses foram armazenados em um banco de dados, no programa estatístico Statistical Package for the Social Sciences (SPSS), versão 20.0, plataforma Windows.

\section{RESULTADOS E DISCUSSÃO}

Foram entrevistados familiares de 100 pacientes internados em duas Unidades de Terapia Intensiva de um hospital público em Feira de Santana - Bahia durante o período do estudo, com média de idade de 49 anos $( \pm 19,55)$ sendo a maioria dos pacientes do sexo masculino $(56 \% ; \mathrm{n}=56)$, admitidos em proporção ligeiramente maior devido a causas clínicas $(57 \% ; \mathrm{n}=57)$ do que cirúrgica $(30 \% ; \mathrm{n}=30)$. Contudo, 13 pacientes (13\%) foram admitidos com diagnósticos clínicos que terminaram por evoluir para cirúrgicos. Foi constatado nesse período internação as principais causas de admissão na UTI foram distúrbios neurológicos $(28 \% ; \mathrm{n}=28)$ o pós-operatório $(22 \% ; \mathrm{n}$ $=22)$, seguido de distúrbios respiratórios $(14 \% ; \mathrm{n}=14)$, politrauma $(11 \% ; \mathrm{n}=11) \mathrm{e}$ distúrbio renal $(10 \% ; \mathrm{n}=10)$.

Dos familiares adscritos no estudo, houve predomínio de familiares habitantes de Feira de Santana (65\%; $n=65)$, a maioria era do sexo feminino $(63 \% ; n=63)$, católica $(57 \% ; \mathrm{n}=57)$, com idade média de 41 anos $( \pm 12,8)$, casada ou com união consensual $(51 \% ; \mathrm{n}=51)$, com escolaridade de ensino médio $(63 \% ; \mathrm{n}=63)$ e com a situação de trabalho atual ativa $(30 \%, \mathrm{n}=30)$. O fato da maioria dos entrevistados serem mulheres, indica o contexto sociocultural e histórico no qual a mulher mantêm o papel de cuidadora, na posição de responsável pelos cuidados prestados à família. (Gutierrez, Minayo, 2010).

Quanto ao grau de parentesco com o paciente internado, os mais frequentes foram filho (a), com $34 \%$ dos casos $(n=34)$, seguido de outro parentesco $(25 \% ; n=25)$ e irmão(ã) $(16 \% ; n=16)$. Do total de familiares, a maioria $(71 \%)$ não mora na mesma residência que o familiar internado e nunca tinha passado pela experiência de ter um parente internado em UTI (71\%).

Quanto ao nível de conforto identificado após aplicação da ECONF, considerando-se a escala de respostas com pontuações que variam de 1 a 5 , sendo uma escala crescente, ou seja, quanto maior a pontuação, maior o nível de conforto relatado. A média do nível global encontrada foi de 3,8 $( \pm 0,5)$ evidenciando que os familiares demonstravam se sentir mais confortáveis do que desconfortáveis. Ao observar o nível de conforto da dimensão Suporte da ECONF através de sua média, verificou-se que o mesmo corresponde a um baixo nível de conforto.

A análise da prevalência de transtornos mentais comum por meio do SRQ-20 revelou um nível de suspeição diagnóstica dos familiares que tinham um parente internado na UTI de 53\%, ou seja, apresentaram rastreamento positivo para transtorno mental comum. Silva et al (2018) investigou a prevalência de transtornos mentais comuns e verificou que varia entre $29,6 \%$ a $47,4 \%$ sendo mais frequente em indivíduos de idade avançada, do sexo feminino, de baixa renda, baixo nível de escolaridade, tabagistas, divorciados ou viúvos, de cor negra ou parda e doentes crônicos, que apresentam maiores prevalências de TMC. 
Foi identificada uma correlação direta fraca entre a categoria Sintomas Somáticos do SRQ-20 e a dimensão Suporte da ECONF ( $r=0,22 ; p=0,03)$. Quanto à categoria Sintomas Somáticos do SRQ-20 observou-se a alta frequência de respostas positivas para tais sintomas. Tais achados denotam que na medida em que aumenta $o$ nível de conforto quantificado pelos familiares, neste estudo, "pouco conforto" relacionado ao suporte, os níveis de sintomas somáticos correspondentes a presença do TMC podem aumentar.

\section{CONSIDERAÇÕES FINAIS (ou Conclusão)}

O presente estudo não apontou correlação negativa entre os escores dos instrumentos, como esperado para confirmar a validade da ECONF, no entanto a análise isolada dos escores dos instrumentos mostrou alta prevalência de transtornos mentais comuns em familiares de pessoas internadas em UTI, e um baixo nível de conforto na dimensão Suporte da ECONF.

Estes achados sugerem que a saúde psíquica dos familiares necessita de atenção. Para isso, é importante o estabelecimento de estratégias de melhor acolhimento destes durante o período de internação do ente nas UTIs e o acompanhamento longitudinal com a equipe de psicologia. Espera-se que esse estudo alerte os profissionais de saúde e as instituições, especialmente os gestores, para as influências que as condutas profissionais e as políticas institucionais rígidas podem ocasionar no estado de saúde mental e bem-estar dos familiares.

\section{REFERÊNCIAS}

JEZIERSKA, N.; BORKOWSKI, B.; GASZYŃSKI, W. Psychological reactions in family members of patients hospitalised in intensive care units. Anaesthesiol Intensive Ther, v. 46, n. 1, p. 42-45, 2014.

PUGGINA, A.C. Percepção da comunicação, satisfação e necessidades dos familiares em Unidade de Terapia Intensiva. Esc. Anna Nery [online]. 2014, vol.18, n.2, pp.277283. ISSN 1414-8145.

FREITAS, K. S.; MUSSI, F. C.; MENEZES, I. G. Desconfortos vividos no cotidiano de familiares de pessoas internadas na UTI. Esc. Anna Nery [online]. 2012, vol.16, n.4, pp.704-711. ISSN 1414-8145.

SILVA, P A S, ROCHA, S V, SANTOS, L B, SANTOS, C A, AMORIM, C R, VILELA, A B A. (2018). Prevalência de transtornos mentais comuns e fatores associados entre idosos de um município do Brasil. Ciência \& Saúde Coletiva, 23(2).

SANTOS, K. O. B.; CARVALHO, F. M.; ARAÚJO, T. M, OLIVEIRA, N. F. Estrutura fatorial e consistência interna do Self-Reporting Questionnaire (SRQ-20) em população urbana. Cad. Saúde Pública [online]. 2009, vol.25, n.1, pp.214-222. ISSN 1678-4464.

SANTOS, K. O. B.; CARVALHO, F. M.; ARAÚJO, T. M. Consistência interna do selfreporting questionnaire-20 em grupos ocupacionais. Revista de Saúde Pública, São Paulo, v. 50, p. 6-, jan. 2016. ISSN 1518-8787.

SANTOS, K. O. B.; ARAÚJO, T. M. D.; PINHO, P. D. S.; SILVA, A. C. C. Avaliação de um instrumento de mensuração de morbidade psíquica: estudo de validação do selfreporting questionnaire (SRQ-20). Revista Baiana de Saúde Pública, 34(3), 2011, 544.

GUTIERREZ, D.M.D.; MINAYO, M.C.S. Produção de conhecimento sobre cuidados da saúde no âmbito da família. Ciência \& saúde coletiva, v.15, p.497-508, 2010.

MOREIRA, E. K. C. B.; MARTINS, T. M.; CASTRO, M. M. Representação social da Psicologia Hospitalar para familiares de pacientes hospitalizados em Unidade de Terapia Intensiva. Rev. SBPH[online]. 2012, vol.15, n.1 [citado 2018-08-13], pp. 134167. 